\title{
Disruption in Circadian Rhythm Increases Cardiovascular Disease Risk Factors in Shift Working Nurses
}

\author{
Ghizal Fatima ${ }^{1}$ Ashish Jha ${ }^{2}$ Mohsin Ali Khan ${ }^{3}$ \\ ${ }^{1}$ Department of Biotechnology, Era's Lucknow Medical College and \\ Hospital, Lucknow, Uttar Pradesh, India \\ 2Department of Cardiology, Ram Manohar Lohiya Institute of \\ Medical Sciences, Lucknow, Uttar Pradesh, India \\ ${ }^{3}$ Era's Lucknow Medical College and Hospital, Lucknow, Uttar \\ Pradesh, India627985
}

Ind J Car Dis Wom 2021;6:79-85.

\begin{abstract}
Keywords

- ambulatory blood pressure monitoring

- circadian rhythm

- day shift work

- night shift work

Background Shift work is associated with behavioral, psychosocial, and physiological consequences in the body that may cause cardiovascular disease (CVD) risk. Therefore, in this study we tried to demonstrate the disruption in circadian rhythm of blood pressure by ambulatory blood pressure monitor in shift working nurses.

Methods A total of 50 nurses, 25 night shift working nurses (NSWN), and 25 day shift working nurses (DSWN) underwent ambulatory blood pressure monitoring (ABPM) for a period of 24 hours. Along with measurements of inflammatory markers (hs-CRP and IL-6) the risk factors for cardiovascular disease between NSWN and DSWN and their possible relationship with disrupted circadian rhythm were measured by circadian rhythm questionnaire.

Results The mean systolic and diastolic pressure for a period of 24 hours and mean diastolic blood pressure analysis throughout the sleep time was found greater in the NSWN than the DSWN $(118 \mathrm{~mm} \mathrm{Hg}$ vs. $112 \mathrm{~mm} \mathrm{Hg}, p<0.05: 72 \mathrm{~mm} \mathrm{Hg}$ vs. $68 \mathrm{~mm}$ $\mathrm{Hg}, p<0.05: 62 \mathrm{~mm} \mathrm{Hg}$ vs. $59 \mathrm{~mm} \mathrm{Hg}, p<0.05)$. High mean blood pressure readings were found to be more frequent in the NSWN $(p<0.05)$ than in DSWN. No significant differences were found in IL- 6 and hs-CRP levels. However, a significant difference in circadian rhythm abnormality was found in NSWN than in DSWN.

Conclusion The 7 day/24 hours ambulatory blood pressure monitoring (ABPM) in NSWN showed a reduced circadian rhythm variation in blood pressure along with abnormality in circadian rhythm itself as compared with DSWN. Work schedules and the consequent rest-activity schedules affect circadian rhythms, with likely long-term impact on health. Therefore, working in critical care unit during night hours leads to abnormal blood pressure in NSWN, suggesting that this type of work, in which sleep is disturbed, leading to mental stress, could be a direct risk factor in cardiovascular diseases.
\end{abstract}

\author{
Address for correspondence Ghizal Fatima, MSC, PhD, Department \\ of Biotechnology, Era's Lucknow Medical College and Hospital, \\ Lucknow, Uttar Pradesh 226017, India \\ (e-mail: ghizalfatima8@gmail.com).
}

DOI https://doi.org/ $10.1055 / \mathrm{s}-0041-1732508$
C 2021. Women in Cardiology and Related Sciences.

This is an open access article published by Thieme under the terms of the Creative Commons Attribution-NonDerivative-NonCommercial-License, permitting copying and reproduction so long as the original work is given appropriate credit. Contents may not be used for commercial purposes, or adapted, remixed, transformed or built upon. (https://creativecommons.org/licenses/by-nc-nd/4.0/).

Thieme Medical and Scientific Publishers Pvt. Ltd. A-12, 2nd Floor, Sector 2, Noida-201301 UP, India 


\section{Abstract Image}

\section{Title: Disruption in circadian rhythm increases cardiovascular disease risk factors in shift working nurses}

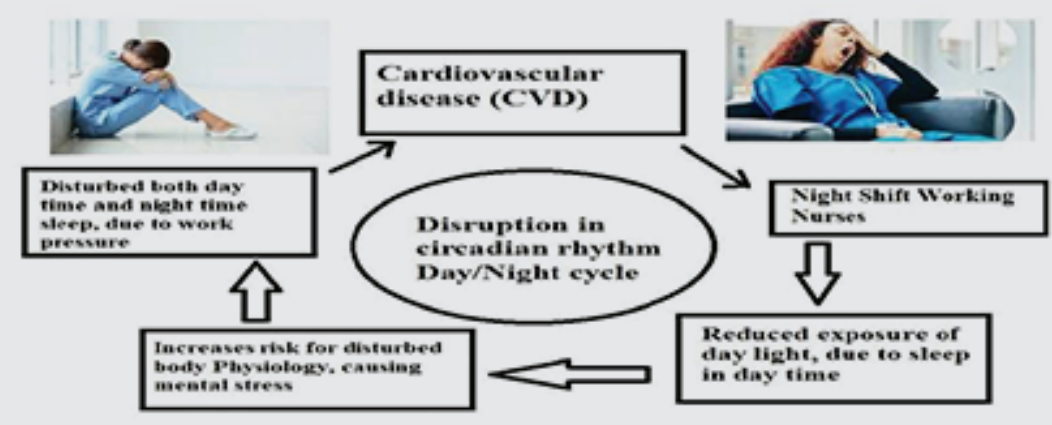

\section{Night shift workers showed a reduced circadian rhythm variation in blood pressure along with abnormality in circadian rhythm itself as compared to day shift workers.}

\section{Introduction}

Shift work does not have a specific definition; whatever work we perform apart from our regular day-to-day working hours is considered a shift work. Shift work, being performed in $\sim 22 \%$ of population in industrialized countries is an essential part of numerous industries. ${ }^{1,2}$ Various forms of illnesses including cardiovascular diseases, diabetes, and obstructive sleep apnea are the consequences of night shift work. ${ }^{3}$ Diseases such as hypertension, left ventricular hypertrophy, coronary heart disease, and myocardial infarction are very common and could be critical in night shift workers than in day shift workers. ${ }^{2}$ All these complications arise as an outcome of the encounter that appears between shifted work hours and the output of the biological clock. ${ }^{4}$ The ability to be synchronized (or entrained) by zeitgebers (external time cues) is a noteworthy feature of circadian rhythm. ${ }^{5}$ For synchronizing endogenous rhythms, light is the most effective stimulus. The time, duration, intensity, and the wavelength of light cause synchronization ${ }^{6}$ via the retinohypothalamic tract to the supra chiasmatic nucleus (SCN) photic information is transmitted in mammals. ${ }^{7}$ Stimuli including scheduled voluntary exercise; food; and exogenous melatonin or serotonergic activation, that is, nonphotic stimuli can additionally shift the endogenous circadian rhythms.
Nocturnal feeding disturbs the synchronization of circadian rhythms and has been shown as a strong entrainer. ${ }^{8}$ A sudden change in the feeding time schedule (from day to night) for some days progressively shifts the phases of the peripheral clocks; it may occur as a result of night shift working hours that directly disrupt circadian rhythm. ${ }^{9}$ When suitably timed, exogenous melatonin shifts the phase of the human circadian clock to the earlier (advance phase shift) or later (delay phase shift) times, having acute sleepiness-inducing and temperature-lowering effects during the biological daytime. ${ }^{10}$

After an unexpected change in an individual's habits and/or environment, a momentary nap throughout the day or a temporary nocturnal awakening that does not trigger a change in the body's endogenous clock may lead to the resynchronization of rhythms. A disparity between the endogenous and exogenous components of the rhythm appears when the difference occurs over a long period ( 5 days). ${ }^{11}$ Poor health is directly linked with this desynchronization of circadian rhythm. Sleep disturbances, shift-lag syndrome, increased risk of errors, and work-related accidents are the short-term consequences of such desynchronization whereas the long-term consequences lead in disturbances of the cardiovascular systems, reproductive difficulties, impaired lipid metabolism, and 
cancer. ${ }^{12}$ Here in this study, we have demonstrated that circadian misalignment increases blood pressure and may affect inflammatory markers. Our findings may help explain why shift working nurses have increased hypertension and cardiovascular disease risk. Therefore, in this study we have measured the disruption in circadian rhythm in night shift working nurses (NSWN).

\section{Materials and Methods}

\section{Subjects Selection and Techniques}

This work was conducted in Era's Lucknow Medical College and Hospitals (ELMCH), Lucknow, India. First, we took the approval from ethics committee of ELMCH; then, identified shift working nurses in critical care unit with their consent to participate in the study were enrolled. Informed consent for the participation in the study was obtained from each participating nurses. Shift working nurses in the age group of 18 to 40 years were included in this study. Nurses with any known comorbid conditions like pre-existing hypertension, coronary artery disease (CAD), rheumatoid arthritis, systemic lupus erythematosus (SLE), diabetes mellitus, and other endocrinal disorder (thyroid and pituitary dysfunction), and psychiatric patients were excluded from the study.

\section{Study Population}

A total of 50 female nurses were screened for inclusion in the study-25 were day shift nurses and 25 were night shift nurses. Complete informed consent was taken from the volunteers willing to participate in the study, and history and medical examination of each subject was recorded. About $4 \mathrm{~mL}$ blood samples were collected from all subjects. Serum was separated for analysis for biochemical markers of cardiovascular diseases.

\section{Measured Variables}

Assessment of Ambulatory Blood Pressure Monitoring When blood pressure (BP) is measured as one move around it is termed as ambulatory blood pressure monitoring (ABPM). For up to 24 hours the BP was measured. A little digital BP monitor was fastened to a belt across the waist and linked to a cuff across the upper arm. The monitor was so compact that one would do the daily chores with it on and may sleep wearing it without getting disturbed. The compact monitor measured the blood pressure analogously as the usual blood pressure monitor does, that is, an electronic monitor records the BP by inflating a cuff across the upper arm and then slowly freeing the pressure. A cover protected the device and it lasted in that for the time period of monitoring. Throughout the day, at regular intervals, the BP reading was recorded, nearly every 15 to 30 minutes in the daytime and 30 to 60 minutes at night. Monitor was placed on nurse's body throughout the night; they were asked not to wear out the device during their sleep. After the monitoring period was over, they could detach the device and cuff, and return it back to the hospital. All the readings were stored in the device, and these were analyzed.

\section{Questionnaires: General Assessment Questionnaire Was Analyzed}

\section{Circadian Rhythm Test (CRT): This Test Was Analyzed Based on Questionnaire \\ Estimation of Serum IL-6 (Geissler et al, 1992)}

For the estimation of serum IL-6, $100 \mu \mathrm{L}$ of standards and samples were added to the plate and six-fold serial dilutions of the $320 \mathrm{pg} / \mathrm{mL}$ top standard was performed in separate tubes. Thus, the human IL-6 standard concentrations were $320 \mathrm{pg} / \mathrm{mL}, 80 \mathrm{pg} / \mathrm{mL}, 40 \mathrm{pg} / \mathrm{mL}, 20 \mathrm{pg} / \mathrm{mL}, 10 \mathrm{pg} / \mathrm{mL}$, and $5 \mathrm{pg} / \mathrm{mL}$. Then it was kept for incubation at $37^{\circ} \mathrm{C}$ for 2 hours. After the incubation time, the plate was aspirated and washed four times with wash buffer, and then dried by firmly tapping it upside down on absorbent paper. Then, $100 \mu \mathrm{L}$ of diluted detection antibody solution was added to each well, and the plate was sealed and kept for incubation at $37^{\circ} \mathrm{C}$ for 1 hour. After the incubation, the plate was washed four times with wash buffer and $100 \mu \mathrm{L}$ of diluted Av-HRP solution was added to each well of the plate and the plate was sealed and kept for incubation at $37^{\circ} \mathrm{C}$ for 30 minutes. Then again the plate was washed four times with wash buffer and the plate wells were soaked in the wash buffer for 30 seconds. Then, $100 \mu \mathrm{L}$ of freshly mixed TMB substrate solution was added in each well of the plate and the plate was incubated in the dark for 30 minutes where the positive wells turned bluish in color. Then the reaction was stopped by adding $100 \mu \mathrm{L}$ of stop solution to each well; the color change was observed and the positive wells turned from blue to yellow. The readings were then taken by keeping the absorbance at $450 \mathrm{~nm}$ within 30 minutes of stopping reaction (hs-CRP was measured by the same pattern of ELISA by the ELISA kit).

\section{Results}

We examined 25 day shift working nurses (DSWN) with mean age $36.7 \pm 9.9$ years and 25 night shift working nurses (NSWN) with mean age of $32.8 \pm 10.5$ years from ELMCH. The 7-day/24-hour blood pressure monitoring recorded in nurses consisted of two groups with day working shifts and night working shift, respectively. During the monitoring, we evaluated the sleep time on different days in every nurse by the sleep questionnaire. Both the group of nurses, the DSWN and the NSWN, were recruited for 7-day ABPM. Medical instruments TM2430 (A\&D, Japan) monitors were used for ABPM (oscillation method). Means of systolic and diastolic BP were evaluated. We calculated mean systolic and diastolic BP for 7 days and for every 24-hour profile. Blood was withdrawn from both the group of nurses after ABPM. Consent was obtained and the ethics committee of our institution gave approval for the study. General assessment questionnaire was filled which contained personal identification variables. The casual BP measurement was performed three 
consecutive times with a validated automatic device with a 2-minute interval between the measurements, with appropriate cuff to the arm size, in the seated position. Presence of at least one of the following possibilities characterized hypertension: usage of antihypertensive drugs, self-reported

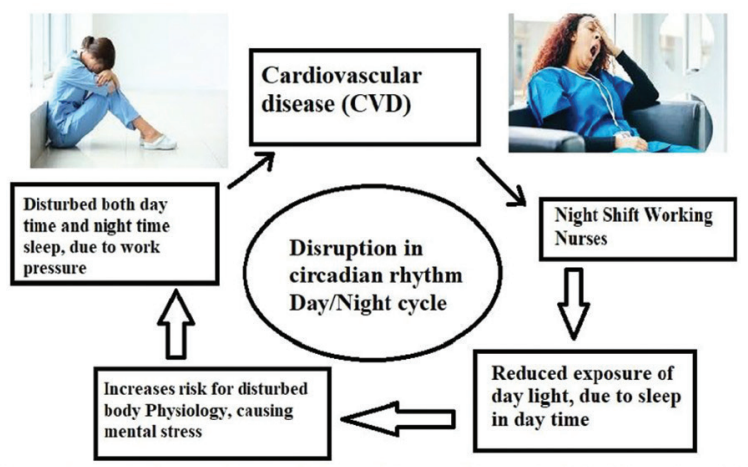

Fig. 1 Disruption in circadian rhythm causes increased risk of cardiovascular diseases in night shift working nurses. hypertension, or the values of the spontaneous measure greater than or equal to $140 \mathrm{~mm} \mathrm{Hg}$ and/or $90 \mathrm{~mm} \mathrm{Hg}$.

ABPM was executed for 24 hours. Based on the schedules described in the participants' activity reports the averages and prevalence of 24-hour hypertension, awake and sleep periods were evaluated. The accepted cutoff points for hypertension for systolic and diastolic pressures, respectively, were $\geq 130 / 80 \mathrm{~mm} \mathrm{Hg}$ in the 24-hour period, $\geq 135 / 85 \mathrm{~mm} \mathrm{Hg}$ in wakefulness, and $\geq 120 / 70 \mathrm{~mm} \mathrm{Hg}$ in the sleep period - Fig. 1 .

\section{Statistical Analysis}

Statistical analysis was performed using the INSTAT 3.0 (Graph Pad Software, San Diego, California, United States). Quantitative variables are presented as the mean \pm standard deviation. Welch's corrected unpaired $t$-test was performed to assess the difference in different parameters among the two groups and the association between clinical characteristics among NSWN and DSWN was expressed by Fisher's exact test, which was used to obtain the odds ratio (OR).

Table 1 Ambulatory blood pressure monitoring in 24-hour period of night shift working nurses (NSWN) and night shift working nurses (DSWN)

\begin{tabular}{|c|c|c|c|c|}
\hline S. No. & Characteristics & NSWN & DSWN & $p$-Value \\
\hline 1 & Length of examination (h) & $24.1 \pm 0.79$ & $24.5 \pm 1.67$ & NS \\
\hline 2 & Adequate reading (\%) & $88.1 \pm 6.97$ & $86.4 \pm 6.87$ & NS \\
\hline 3 & Total readings & $72.7 \pm 7.17$ & $69.9 \pm 8.89$ & $<0.05$ \\
\hline 4 & Duration of sleep (min) & $250.8 \pm 65.21$ & $431.8 \pm 63.55$ & $<0.05$ \\
\hline 5 & Awake duration reading & $60.7 \pm 6.97$ & $49.9 \pm 10.42$ & $<0.05$ \\
\hline 6 & Sleep duration reading & $12.9 \pm 3.60$ & $18.9 \pm 4.6$ & $<0.05$ \\
\hline 7 & 24-hour systolic blood pressure (\%) & $5.8 \pm 8.90$ & $4.5 \pm 7.8$ & NS \\
\hline 8 & 24-hour diastolic blood pressure (\%) & $12.9 \pm 16.01$ & $9.3 \pm 11.12$ & NS \\
\hline 9 & Awake time systolic blood pressure (\%) & $2.6 \pm 5.35$ & $3.1 \pm 6.39$ & NS \\
\hline 10 & Awake time diastolic blood pressure (\%) & $7.2 \pm 11.21$ & $6.1 \pm 8.90$ & NS \\
\hline 11 & Sleep time systolic blood pressure (\%) & $3.4 \pm 8.01$ & $2.7 \pm 5.90$ & NS \\
\hline 12 & Sleep time diastolic blood pressure (\%) & $1.9 \pm 4.31$ & $1.8 \pm 3.33$ & NS \\
\hline 13 & Pulse pressure $(\mathrm{mm} \mathrm{Hg})$ & $44.6 \pm 4.87$ & $45.3 \pm 4.35$ & NS \\
\hline
\end{tabular}

Abbreviation: NS, not significant.

Table 2 Ambulatory blood pressure monitoring in day shift working nurses (DSWN) and night shift working nurses (NSWN): mean reading of blood pressure (BP) in $\mathrm{mm} \mathrm{Hg}$

\begin{tabular}{|l|l|l|l|l|}
\hline S. No. & BP & NSWN & DSWN & $p$-Value \\
\hline 1 & 24-hour systolic BP & $118 \pm 7.58$ & $112 \pm 7.61$ & $<0.05$ \\
\hline 2 & 24-hour diastolic BP & $72 \pm 5.83$ & $68 \pm 5.95$ & $<0.05$ \\
\hline 3 & Systolic sleep BP & $105 \pm 8.42$ & $102 \pm 8.42$ & NS \\
\hline 4 & Diastolic sleep BP & $62 \pm 5.89$ & $59 \pm 6.44$ & $<0.05$ \\
\hline 5 & Systolic awake BP & $118 \pm 7.80$ & $116 \pm 7.82$ & NS \\
\hline 6 & Diastolic awake BP & $75 \pm 6.12$ & $72 \pm 6.15$ & NS \\
\hline
\end{tabular}

Abbreviation: NS, not significant.

Note: Data presented as mean \pm standard deviation (SD). 
Table 3 Clinical and biochemical characteristics of day shift working nurses (DSWN) and night shift working nurses (NSWN)

\begin{tabular}{|l|l|l|l|}
\hline Variables & $\begin{array}{l}\text { NSWN } \\
n=25 \\
\text { Mean } \pm \text { SD (\%) }\end{array}$ & $\begin{array}{l}\text { DSWN } \\
n=25 \\
\text { Mean } \pm \text { SD (\%) }\end{array}$ & $p$-Value \\
\hline ESR & $27.2 \pm 9.7$ & $24.9 \pm 8.2$ & NS \\
\hline ALT & $39.8 \pm 14.1$ & $37.6 \pm 14.4$ & NS \\
\hline IL-6 & $2.6 \pm 2.1$ & $2.2 \pm 1.4$ & NS \\
\hline hs-CRP & $3.0 \pm 2.4$ & $2.3 \pm 1.7$ & NS \\
\hline Frequent awakening & $10(40)$ & $2(8)$ & $<0.05$ \\
\hline Disturbed sleep & $12(48)$ & $4(16)$ & $<0.05$ \\
\hline Headache & $13(52)$ & $2(8)$ & $<0.05$ \\
\hline Abdominal pain & $4(16)$ & $0(0)$ & NS \\
\hline Lack of energy & $19(76)$ & $1(4)$ & $<0.05$ \\
\hline
\end{tabular}

Abbreviations: ESR, ...; ALT, ...; IL-6, ...; hs-CRP, ...; NS, not significant; SD, standard deviation.

Note: $p<0.05$ is considered significant, $p<0.01$ is considered highly significant, and NS is considered not significant by chi-square and unpaired $t$-test.

Table 4 Circadian rhythm test in patients and controls

\begin{tabular}{|c|c|c|c|}
\hline Circadian rhythm condition & $\begin{array}{l}\text { NSWN } \\
n=25(\%)\end{array}$ & $\begin{array}{l}\text { DSWN } \\
n=25(\%)\end{array}$ & $p$-Value \\
\hline Misaligned body clock & $13(54)$ & $2(8)$ & \multirow[t]{4}{*}{$<0.05$} \\
\hline Non body clock related & $4(16)$ & $0(0)$ & \\
\hline Body clock amplitude disorder & $2(8)$ & $0(0)$ & \\
\hline Normal body clock & $6(24)$ & $23(92)$ & \\
\hline \multicolumn{4}{|l|}{ Circadian rhythm severity } \\
\hline Mild & $5(20)$ & $0(0)$ & \multirow[t]{4}{*}{$<0.05$} \\
\hline Moderate & $12(48)$ & $2(8)$ & \\
\hline Severe & $2(8)$ & $0(0)$ & \\
\hline No abnormality & $6(24)$ & $23(92)$ & \\
\hline
\end{tabular}

Abbreviations: DSWN, day shift working nurses; NSWN, night shift working nurses.

Note: $p<0.05$ is considered highly significant by chi-square test

All statistical tests were two-tailed, and $p<0.05$ was chosen as the level of significance.

\section{Ambulatory Blood Pressure Monitoring in NSWN and DSWN}

- Table 1 shows the changes and the significant levels in total readings of ABPM in NSWN and DSWN. Significant differences were observed in duration of sleep (in minutes); awake duration reading and sleep duration reading depicted significant changes in both the groups. However, no such differences of significance were observed in the rest of the parameters. Table2 clearly depicts the picture of abnormality in NSWN and DSWN in the mean BP of 24-hour systolic and 24-hour diastolic BP monitoring along with disturbances in diastolic sleep BP rhythm by measurement from ABPM, resulting in clear disturbances in BP leading to cardiovascular diseases.

\section{Clinical and Biochemical Characteristics of NSWN and DSWN}

The clinical and biochemical characteristics of NSWN and DSWN are shown in - Table 3 . In the clinical assessment of
NSWN and DSWN groups, frequent awakening, sleep status, headache, abdominal pain, and lack of energy are all taken from the questionnaire part where frequent awakening, disturbed sleep, headache, and lack of energy were more commonly seen in NSWN than in DSWN. However, the clinical characteristics were not found significant in both the group of nurses.

\section{Circadian Rhythm Test in NSWN and DSWN}

Circadian rhythm test by Philips questionnaire was done in 25 DSWN and 25 NSWN in which 13 (52\%) NSWN and 2 (8\%) DSWN showed a misaligned body clock. Non body clock related was seen in 4 (16\%) NSWN. Further, body clock amplitude disorder was seen in 1 (4\%) NSWN and $0(0 \%)$ DSWN, and normal body clock condition was found in 8 (32\%) NSWN and 23 (92\%) DSWN. In the analysis of circadian rhythm severity 5 (20\%) NSWN and $0(0 \%)$ controls were reported to be mild and 12 (48\%) NSWN and 2 (8\%) DSWN fell in the category of moderate. While 2 ( $8 \%$ ) NSWN reported to be in the severe category, and no DSWN were in severe, no abnormality was found in 6 NSWN whereas 23 DSWN had no abnormality of circadian rhythm severity score ( - Table 4 ). 


\section{Discussion}

Shift work is any work that exceeds more than 8 hours of regular working period, therefore interfering with the natural cycle of biological rhythm of our body. Workers in night shift have a greater risk for CVD in comparison to an individual working normal hours; night time shift working is more hazardous to health as it directly impacts the biological and physiological system of the body. However, the exact mechanism of the impact of shift work on cardiovascular health is yet to be understood. BP modifications may be a possible factor in that nocturnal work modifies the circadian rhythm of $\mathrm{BP}$ and those individuals in whom there is a fall in nocturnal BP (dippers) become nondippers when they start working at night time. In a single day of working in night hours there is a high systolic BP level. ${ }^{13}$

In this article, we examined 25 DSWN with mean age 36.7 \pm 9.9 years and 25 NSWN with mean age $32.8 \pm 10.5$ years. The 7-day/24-hour BP monitoring recorded in nurses consisted of two groups with DSWN and NSWN. During the monitoring, we evaluated the sleep time on different days in every nurse using the sleep questionnaire. Both groups of nurses, the DSWN and the NSWN, were recruited for 7-day ABPM.

An integral part in sleeping time differences between the NSWN and DSWN was also observed by the questionnaire. As in NSWN there is already a lack of rest activity that is very important for our health and not resting properly directly elevates catecholamine, resulting in interference in normal BP. NSWN were not different from the DSWN. Maybe because the similar loads in BP can represent different mean readings of $B P$, we, therefore, could not find any difference in that in our study. More than $53 \mathrm{~mm} \mathrm{Hg}$ pulse pressure is also related to a greater risk of CVD. ${ }^{14}$ Perhaps, because we had more young and healthy individuals in our study, no difference was found in the pulse pressure readings in both the groups.

In NSWN, the mean BP was higher, although in statistics the difference was recorded only in the systolic and diastolic 24-hour BP and the diastolic BP. Our findings are in agreement with other publications. ${ }^{15}$ Moreover, a study on nurses working in night found lower systolic BP and average readings of diastolic BP. However, in that study, the comparison was done on the basis of daytime reading of BP for day workers and nighttime reading of BP for night workers. ${ }^{16}$

During the 24 hours of night shift work more frequent high abnormal mean reading of BP was observed. This study is very similar to a study that was conducted on 62 doctors for evaluating BP with an ambulatory blood pressure monitor. In that study it was observed that only 3 patients in the 24-hour period had abnormally high mean BP readings, whereas 6 and 9 patients during the work period had high mean systolic BP and diastolic BP readings. ${ }^{17}$ It was suggested through study that such cases could resemble "masked hypertension," that is, a condition where the BP levels are normal in office but rise when measured with ambulatory blood pressure monitor. ${ }^{18}$

A study by Bevan et al showed 16 junior medical staff in a 24-hour shift work in the emergency room and in common working day and found higher nocturnal systolic BP and diastolic BP readings. It also found lower nocturnal BP fall during the 24-hour night shift work. ${ }^{19}$ Another study with 37 residents reported higher BP loads and less night time BP fall during 24-hour shift work. ${ }^{20}$ In contrast to our study, these studies used a fixed time period to describe sleep and awake periods without corrections for the real period of wakefulness and sleep, which probably led to higher nocturnal BP readings and consequently to less nocturnal BP fall. These results also found higher mean BP readings during the 24-hour shift work, including values considered as hypertensive in few or some periods.

During the last few years, many studies have been published dealing in different works of many genetics and biochemical parameters studied in cardiovascular diseases. This study is, however, the most extensive so far to look into the relation between the NSWN and DSWN and cardiovascular alterations, mainly BP levels in young nurses in critical care unit, by analyzing ABPM and the circadian rhythm questionnaire. Further, in this study we observed that nurses working in night shifts in the critical care have higher mean 24-hour systolic, diastolic, and sleeping time diastolic BP levels compared with those working during the day time. This may happen because of lack of sleep, and lack of sleep directly elevated mental stress. Moreover, mean BP levels above the normal values, especially the 24-hour diastolic BP and BP levels in all time periods, were related to 24 -hour shift work in the critical care.

\section{Conclusion}

The 7-day/24-hour ABPM in NSWN showed reduced circadian rhythm variations in BP as compared with DSWN. Work schedules and the consequent rest-activity schedules affect circadian rhythms, with likely long-term impact on health. Therefore, working in the critical care unit during the night hours leads to abnormal BP in NSWN, suggesting this type of work, in which sleep is disturbed leading to mental stress, could be a direct risk factor in cardiovascular diseases.
Audio
Audio file for this article is available at https://doi. org/10.1055/s-0041-1732508.

\section{Conflicts of Interest}

None declared.

\section{References}

1 Lee S, McCann D, Messenger JC, Working Time Around the World: Trends in Working Hours, Laws and Policies in a Global Comparative Perspective. London and Geneva: Routledge and ILO; 2007 
2 Haus E, Smolensky M. Biological clocks and shift work: circadian dysregulation and potential long-term effects. Cancer Causes Control 2006;17(4):489-500

3 Persson HE, Svanborg E. Sleep deprivation worsens obstructive sleep apnea. Comparison between diurnal and nocturnal polysomnography. Chest 1996;109(3):645-650

4 Akerstedt T. Shift work and disturbed sleep/wakefulness. Occup Med (Lond 2003;53(2):89-94

5 Vitaterna MH, Takahashi JS, Turek FW. Overview of circadian rhythms. Alcohol Res Health 2001;25(2):85-93

6 Scheer FA, Wright KP Jr, Kronauer RE, Czeisler CA. Plasticity of the intrinsic period of the human circadian timing system. PLoS One 2007;2(8):e721

7 Gooley JJ, Lu J, Chou TC, Scammell TE, Saper CB. Melanopsin in cells of origin of the retinohypothalamic tract. Nat Neurosci 2001;4(12):1165

8 Minors DS, Waterhouse JM. Does 'anchor sleep' entrain circadian rhythms? Evidence from constant routine studies. J Physiol 1983;345:451-467

9 Hirota T, Fukada Y. Resetting mechanism of central and peripheral circadian clocks in mammals. ZoolSci 2004;21(4):359-368

10 Arendt J, Skene DJ. Melatonin as a chronobiotic. Sleep Med Rev 2005;9(1):25-39

11 Waterhouse J. Jet-lag and shift work: (1). Circadian rhythms. J R Soc Med 1999;92(8):398-401

12 Boivin DB, Tremblay GM, James FO. Working on atypical schedules. Sleep Med 2007;8(6):578-589
13 Adams SL, Roxe DM, Weiss J, Zhang F, Rosenthal JE. Ambulatory blood pressure and Holter monitoring of emergency physicians before, during, and after a night shift. AcadEmerg Med 1998;5(9):871-877

14 Verdecchia P. Prognostic value of ambulatory blood pressure: current evidence and clinical implications. Hypertension 2000; 35(3):844-851

15 Schnall PL, Pieper C, Schwartz JE, et al. The relationship between "job strain," workplace diastolic BP, and left ventricular mass index. JAMA 1990;263:1929-1935

16 Munakata M, Ichi S, Nunokawa T, et al. Influence of night shift work on psychologic state and cardiovascular and neuroendocrine responses in healthy nurses. Hypertens Res 2001;24(1):25-31

17 Yetman RJ, West MS, Portman RJ. Changes in circadian rhythm of blood pressure in on-call pediatric residents. ChronobiolInt 1994;11(1):54-61

18 Ohkubo T, Kikuya M, Metoki H, et al. Prognosis of "masked" hypertension and "white-coat" hypertension detected by $24-\mathrm{h}$ ambulatory blood pressure monitoring 10-year follow-up from the Ohasama study. J Am Coll Cardiol 2005;46(3):508-515

19 BevanEG, Findlay JG, Murray GD, McInnes GT. Twenty-four-hour blood pressure in junior medical staff. J R Coll Physicians Lond 1992;26(4):367-371

20 Yeatman RJ, West MS, Portman RJ. Changes in circadian rhythm of BP in on-call pediatric residents. ChronobiolInt 2018;11:54-61 